\title{
Parylene Coated Silicon Probes for Neural Prosthesis
}

\author{
Ray Huang ${ }^{1 *}$, Changlin Pang ${ }^{1}$, Yu-Chong Tai ${ }^{1}$, Jeremy Emken ${ }^{2}$, Cevat Ustun ${ }^{2}$, Richard Andersen ${ }^{2}$ \\ ${ }^{1}$ Micromachining Laboratory, California Institute of Technology, USA \\ ${ }^{2}$ Biology Division, California Institute of Technology, USA
}

\begin{abstract}
Silicon neural prosthetic probes require reliable sensing electrodes able to access deep cortical structures without breakage. However, manufacturing limitations have prevented a strong and biocompatible silicon electrode array from reaching this goal. We here demonstrate the first high-density, parylene-coated silicon probe $(1.2 \mathrm{~cm}$ long) with micro-fabricated electrodes that is able to be inserted in vivo without failure. This work also presents new experimental results for array shank deflection testing, lifetime soak testing as well as the in vitro electrical characterization of the gold and platinum micro-electrodes. These results allow us to optimize the geometry and treatment for both the silicon probe and the metal electrodes.
\end{abstract}

Keywords - parylene cable; neural prosthesis; silicon probe

\section{INTRODUCTION}

An important component of silicon neural prosthetic is an electrode array capable of recording neural activity from the cortex. Such an array should be biocompatible and possess both strong mechanical properties to overcome insertion forces and the ability to accommodate a high number of electrodes with optimal recording characteristics. However, strong mechanical properties and higher electrode densities are often associated with larger geometries which may cause more cortical damage during insertion. Thus, a biocompatible platform that balances the size and signal acquisition capability tradeoffs must be studied and developed.

In this work, we present a total parylene coated silicon probe that has the potential to overcome these difficulties. Two major improvements of the current device over its predecessor [1] are the complete encapsulation of the array with parylene and the smoothing of the sidewalls of the silicon shanks by meniscus etching. Our previous generation consisted of a silicon substrate that was exposed on all sides except the front side, significantly reducing the biocompatibility of the device. Aside from biocompatibility concerns, an untreated array could be more prone to breakage due to surface defects created during deep reactive ion etching (DRIE). As before, we have chosen to use parylene C (polypara-xylylene C) due to its insulation capability, flexibility, biocompatibility, and its prior use in medical applications.

Regarding recording capability, an ideal metal electrode for neural prosthetics application would have low impedance and high spatial specificity with a high signal-to-noise ratio (SNR). However, low impedance and high specificity are competing attributes as low impedances allow the electrode to pick up a larger number of neurons. Also, impedance and area, and thus specificity, are inversely correlated, making it difficult to optimize both SNR and specificity.

This work further introduces the characterization of platinum and gold micro-electrodes. The electrode's impedance dependency on the size of the plasma-etch-opened metal electrodes is presented, which will allow us to determine the optimum material and size of the electrode for neuron specificity testing as well as future electrode optimization for primate prosthesis recording.

As a result, we are able to fabricate silicon probe devices that are well characterized, fully biocompatible, and easy to mass fabricate which will facilitate future research in developing a neural prosthetic system.

\section{DEVICE DESIGN}

A flexible parylene lift-off technology [2] allows us to fabricate 2-D 32 channel flexible cabled electrode array devices. It is a major feature of our device that the cabling is not a component that is later attached to the array but that it is an integrated extension of the top parylene structure of the array itself. These devices can be expanded to 3-D $32 \mathrm{~N}$ channel structures by probe stacking $\mathrm{N}$ number of $2 \mathrm{D}$ probes. Figure 1 shows an unpackaged cabled silicon probe. The circular platinum rings arranged in a $60^{\circ} \mathrm{Y}$ shape pattern on the end of device are used to electrically bond to commercial available connectors with conductive epoxy on a circular PC board.

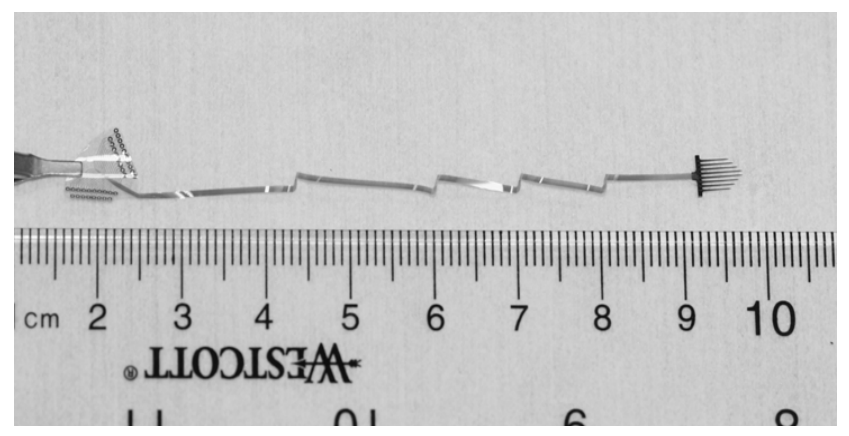

Figure 1. Released silicon probe with parylene flexible cable designed for rat in vivo acute testing. Cable length is approximately $7 \mathrm{~cm}$ and the silicon shank length is approximately $5 \mathrm{~mm}$.

In order to test the mechanical properties of the new silicon probes in vitro and in vivo, test probes are fabricated (Figure 2). The shank lengths range from $10 \mathrm{~mm}$ to $12 \mathrm{~mm}$ and are designed to reach deep cortical regions. Shanks are $150 \mu \mathrm{m}$

This project was funded by the Defense Advanced Research Projects Agency (DARPA) (Award \# 908165) and by National Institute of Health (NIH) moveable probe project (Award \# R01EY015545)

* Contacting Author: Ray Huang, Caltech Micromachining Lab, California Institute of Technology, Pasadena, CA 91125, USA. phone:+1-626-395-2227;fax:+1-626-584-9104;

email:rhuang@caltech.edu 
thick and $60 \mu \mathrm{m}$ wide towards the tip of the shank, which forms a $10^{\circ}$ angle to facilitate insertion into the brain.

Impedance to area mapping of metal electrodes is essential for the optimization of the recording sites. An electrode only flexible parylene cable device is designed for this purpose (Figure 3). Both gold and platinum were used to fabricate 30 electrodes on each device with fifteen different areas that range from $32 \mu \mathrm{m}^{2}$ to $3400 \mu \mathrm{m}^{2}$. Each of the electrodes has a side wall height of $6.5 \mu \mathrm{m}$.

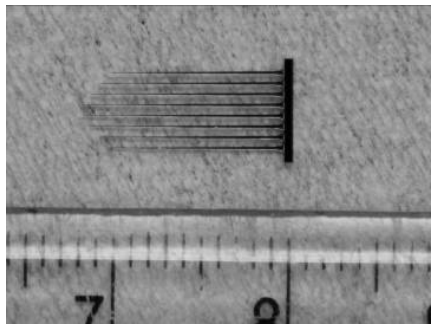

Figure $2.1 .2 \mathrm{~cm}$ silicon probe shanks designed specifically to reach human / primate deep cortical structures. It is used in this work for in vitro bending and in vivo insertion experimentation.

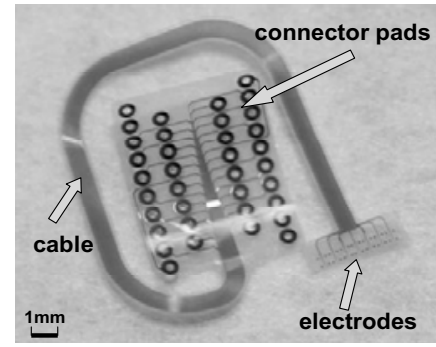

Figure 3. Testing device for electrode impedance mapping experiment. There is no silicon shank attached at the bottom of the electrodes

\section{FABRICATION}

The high density silicon probes are fabricated on double side polished wafers with DRIE technology. Figure 4 shows the fabrication process [1]. First, $0.5 \mu \mathrm{m}$ of parylene $\mathrm{C}$ is deposited on one side of the double side wafer and is melted in an oven at $350^{\circ} \mathrm{C}$, which is then patterned by oxygen plasma to leave areas for adhesion enhancement between the parylene / silicon interface. A sacrificial photoresist layer of $1 \mu \mathrm{m}$ thickness is then coated to facilitate the final release of the device. A bottom layer of parylene $\mathrm{C}(6.5 \mu \mathrm{m})$ is then deposited. This is followed by a layer of lift-off electron-beam evaporated platinum $(0.25 \mu \mathrm{m})$ to define the trace lines, the electrodes and connector pads. The top layer of parylene $\mathrm{C}$ $(6.5 \mu \mathrm{m})$ is deposited to complete the parylene-metal-parylene sandwich structure. Electrode sites and the device definition are then etched by a two step reactive ion etching (RIE) with $\mathrm{O}_{2}$ Plasma process. Silicon probe shanks are subsequently defined and etched by DRIE from both sides of the wafer. The bottom DRIE etching duration defines the thickness of the probe; by depositing and painting protective photoresist die by die, the thickness of every probe on the wafer can be controlled precisely. The devices are then released in photoresist stripper and polished by meniscus etching [3], which is performed by submersing the probe in 1:19/ $\mathrm{HF}: \mathrm{HNO}_{3}$ silicon etchant and gradually pulling it out with a micro-manipulator (Figure 5) at approximately $1 \mathrm{~mm}$ per minute. The result of the etching is shown in figure 6 .

The silicon probes and cable are then totally coated on all sides. This is done by covering the connector pads with handpainted photoresist and conformal coating $1 \mu \mathrm{m}$ of parylene $\mathrm{C}$ over the entire device. The device is covered with photoresist again to protect the cables and the rest of the device, leaving the silicon probe section exposed. This structure is then etched directionally with RIE to re-open the parylene coated electrodes (Figure 7). This device is later released in acetone and photoresist stripper solution. The silicon probe was dipped into a silicon etchant $\left(14 \mathrm{CH}_{3} \mathrm{COOH}+10 \mathrm{HF}+5 \mathrm{HNO}_{3}\right.$ [4]) after the RIE etching to test for total coating. The silicon was not etched after 1 minute of dipping, which validates the coating is still intact and thus full biocompatibility is achieved. (Figure 8) Finally, the device is annealed at $200^{\circ} \mathrm{C}$ for two days to bond the sandwiching parylene layers.
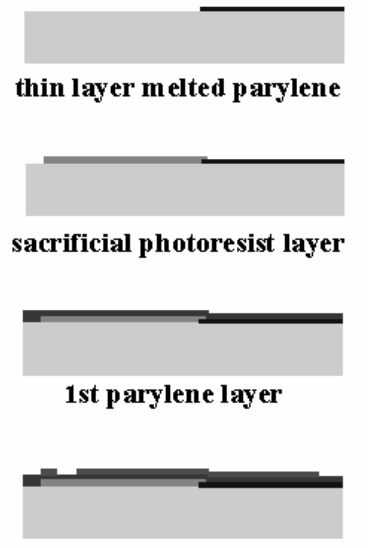

platinum trace lines

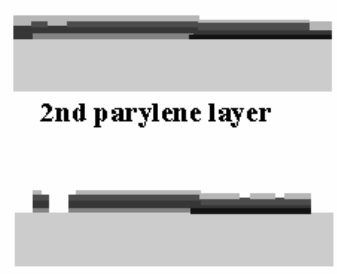

RIE open electrode and define structure

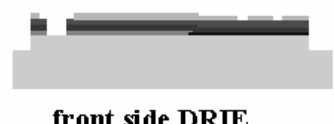

front side DRIE

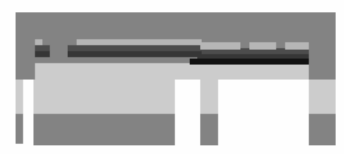

back side DRIE

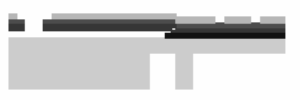

release structure

ㅂ.

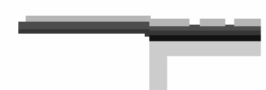

isolate probe and m eniscus etching

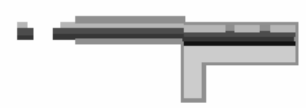

conformal coating

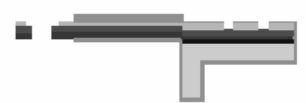

re-open electrode by RIE

Figure 4. Fabrication process steps of the complete device 


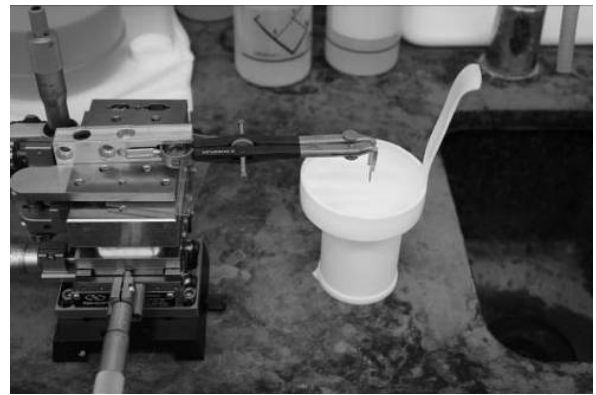

Figure 5. Meniscus etching setup. The probe-only device is pulled out of the silicon etchant by a manipulator located on the left hand side of the picture.
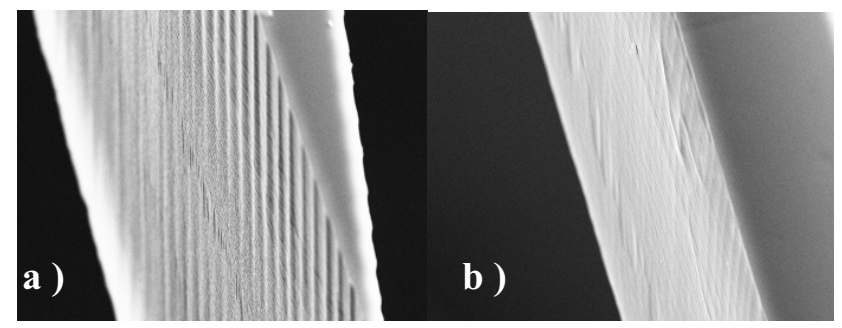

Figure 6. a) Silicon probe before meniscus-etching b) silicon probe after meniscus-etching. The etching rate is estimated to be around $7 \mu \mathrm{m} /$ minute.

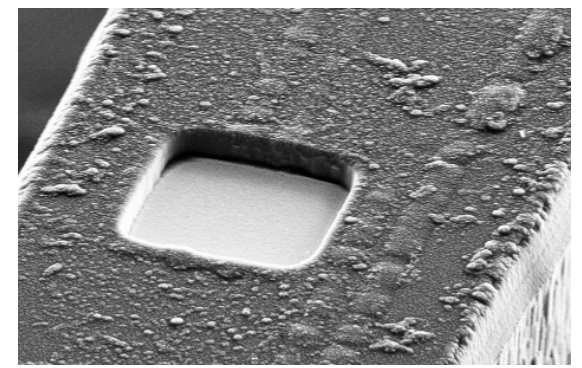

Figure 7. Opened electrodes after final RIE etching

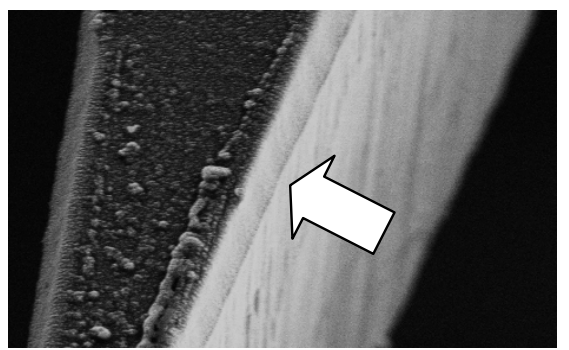

Figure 8. Silicon probe edge after final RIE etching. The edge is still sealed after etching.

\section{RESULT AND DISCUSSION}

Device testing was performed in four phases: mechanical testing of the silicon shanks, in-vivo probe penetration testing, life time testing of the silicon probes and metal electrode characterization.

In the first phase, we used the set up in (Figure 9) to conduct the mechanical deflection testing of the shank only devices. Four types of sample with four devices each $(\mathrm{N}=4)$ were used to observe the effect of each treatment. Table 1 shows that meniscus-etching and parylene coating both improved the strength of the arrays for all bending directions. A parylene coated, meniscus-etched shank with length $1.2 \mathrm{~cm}$ is able to safely deflect around $5.4 \mathrm{~mm}$ from top bending and more than $3.8 \mathrm{~mm}$ from side bending before breaking (Figure 10). Shanks are also consistently breaking further away from the tip after treatment showing the elimination of defects along the shanks. This improvement both reduces the chance of breakage of the device during handling/insertion and reduces probe residues left in test subjects in case of breakage.

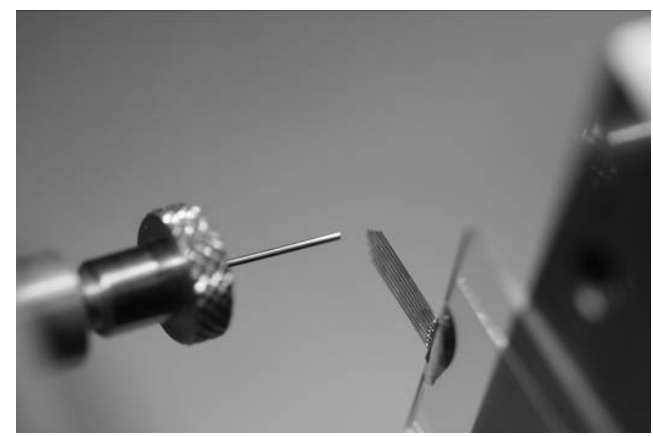

Figure 9. Silicon probe deflection test setup. The silicon probe (right hand side) is glued to a glass slide by epoxy and deflected by a metal rod attached to a force gauge.

TABLE I. SAMPLES WITH COMBINATION OF FACTORS AND TEST RESULT. SAMPLE 1 HAS $200 \mu \mathrm{M}$ BASE WIDTH AND THICKNESS OF $175 \mu \mathrm{M}$. 4 DEVICES

\begin{tabular}{|c|c|c|c|c|}
\hline $\begin{array}{l}\text { Sample } \\
\text { Number }\end{array}$ & $\begin{array}{c}\text { Parylene } \\
\text { Total Coated }\end{array}$ & Sharpened & $\begin{array}{l}\text { Top Deflection } \\
\text { / Force }\end{array}$ & $\begin{array}{l}\text { Top Breaking } \\
\text { point (away from } \\
\text { tip) }\end{array}$ \\
\hline 1 (controlled) & & & $5.36 \mathrm{~mm} / 71 \mathrm{mN}$ & $10 \mathrm{~mm}$ \\
\hline 2 & & $x$ & $5.69 \mathrm{~mm} / 65 \mathrm{mN}$ & $9.69 \mathrm{~mm}$ \\
\hline 3 & $\mathrm{x}$ & & $5.45 \mathrm{~mm} / 70 \mathrm{mN}$ & $11.56 \mathrm{~mm}$ \\
\hline \multirow[t]{2}{*}{4} & $\mathrm{x}$ & $\mathrm{x}$ & $5.40 \mathrm{~mm} / 80 \mathrm{mN}$ & $11.87 \mathrm{~mm}$ \\
\hline & $\begin{array}{l}\text { Side Deflection / } \\
\text { Force }\end{array}$ & $\begin{array}{c}\text { Side Breaking } \\
\text { point (away from } \\
\text { tip) }\end{array}$ & $\begin{array}{l}\text { Bottom Defl. } \\
\text { / Force }\end{array}$ & $\begin{array}{l}\text { Bottom Breaking } \\
\text { point (away from } \\
\text { tip) }\end{array}$ \\
\hline 1 (controlled) & $3.05 \mathrm{~mm} / 47 \mathrm{mN}$ & $3.6 \mathrm{~mm}$ & $1.77 \mathrm{~mm} / 34 \mathrm{mN}$ & $8.6 \mathrm{~mm}$ \\
\hline 2 & $3.10 \mathrm{~mm} / 52 \mathrm{mN}$ & $3.8 \mathrm{~mm}$ & $3.02 \mathrm{~mm} / 61 \mathrm{mN}$ & $8.95 \mathrm{~mm}$ \\
\hline 3 & $3.54 \mathrm{~mm} / 54 \mathrm{mN}$ & $5.08 \mathrm{~mm}$ & $1.97 \mathrm{~mm} / 30 \mathrm{mN}$ & $11.69 \mathrm{~mm}$ \\
\hline 4 & $3.81 \mathrm{~mm} / 70 \mathrm{mN}$ & $5.2 \mathrm{~mm}$ & $2.51 \mathrm{~mm} / 49 \mathrm{mN}$ & $11.44 \mathrm{~mm}$ \\
\hline
\end{tabular}

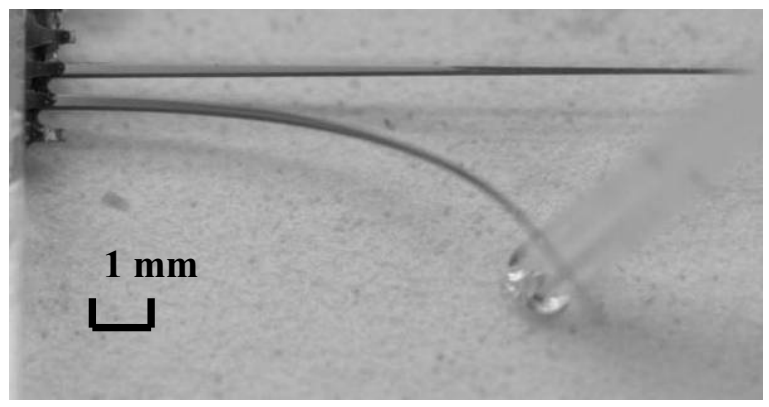

Figure 10. Silicon probe bending from the side by hand

Secondly, the devices were inserted through the pia and into the cortex of rats to test penetration ability. Results show a full 
insertion of the probe was successful without any bending, buckling or breakage by observing the shanks under a surgical microscope during insertion. Cortical dimpling induced by the array during this in vivo insertion was estimated to be approximately $1 \mathrm{~mm}$.

In the third phase, the devices underwent accelerated life testing in saline to determine the mean time to failure of the silicon to parylene adhesion. Soaking test results show that the parylene-silicon adhesion is able to withstand more than five weeks in $90^{\circ} \mathrm{C}$ saline (Figure 11) before delamination occurs.

Finally, device testing of the metal electrodes was conducted by submersing the electrode only structure in saline solution and measuring the impedance values at $1 \mathrm{kHz}$. We tested six devices $(\mathrm{N}=6)$. It is observed that platinum electrodes have impedance values significantly lower than those of the gold electrodes, allowing higher specificity for the same electrode area (Figure 12). The result also shows a linear relationship in log-log scale plot of impedance vs. area and impedance vs. frequency. (Figure 13)

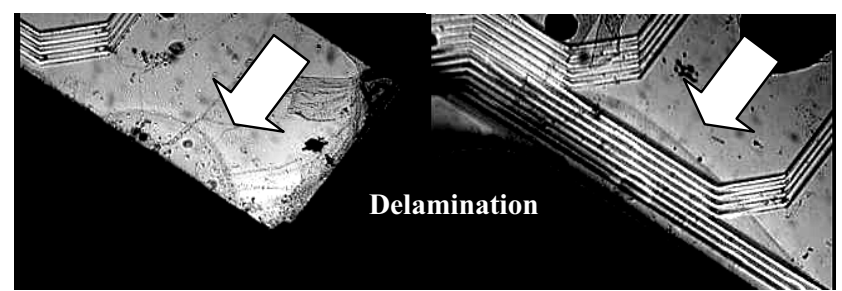

Figure 11. Delamination occurrence after five weeks of soaking in $90^{\circ} \mathrm{C}$ saline solution. The probe was still attached on the parylene cable after eight weeks of soaking.

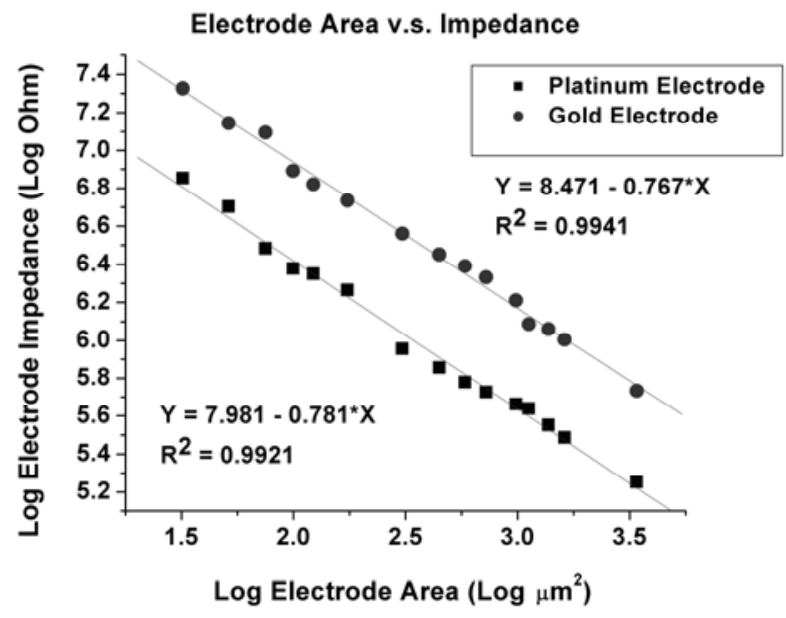

Figure 12. Log-log scale plot of gold and platinum electrode impedance mapping as a function of area measured at $1 \mathrm{kHz}$.

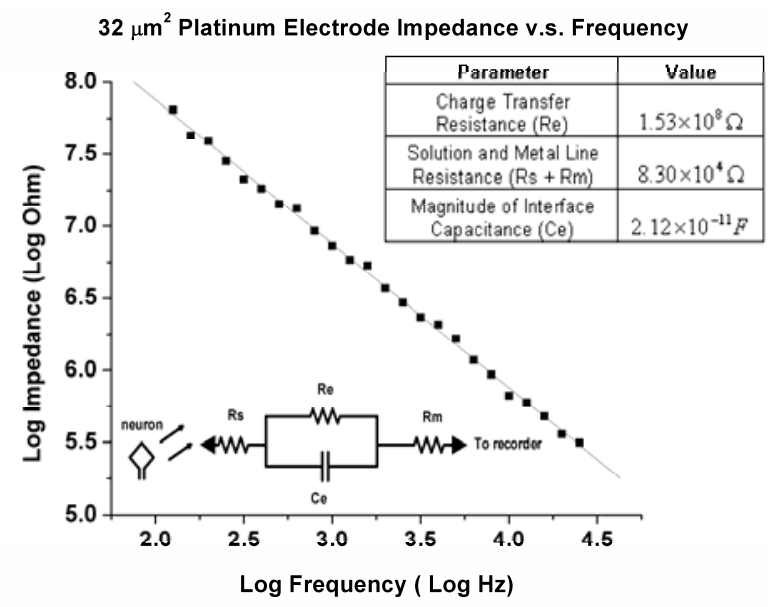

Figure 13. Log-log scale plot of impedance vs. frequency. The legend shows the fitted values of the components in the equivalent circuit model [5].

\section{CONCLUSION}

We have developed and demonstrated the feasibility of a fully parylene $\mathrm{C}$ encapsulated, high electrode density, silicon based array with an integrated flexible cable that is able to access deep cortical structures. The result of the mechanical deflection test was presented and the in vivo insertion capability of the silicon probes has been verified. High temperature accelerated life time testing in saline indicates the stability of parylene-silicon interface for more than five weeks in $90^{\circ} \mathrm{C}$ saline. The area-impedance mapping of platinum and gold electrodes was completed, which will facilitate the optimization of the electrode recording performances. From the testing results, this device shows promise as a neural probe platform to be incorporated into a future chronic cortical prosthetic. Future work is underway to optimize the electrode placement, geometry and platinum black coating process for acute signal specificity testing.

\section{ACKNOWLEDGMENT}

The authors would also like to thank Mr. Trevor Roper, other members of the Caltech Micromachining Laboratory and Andersen lab at Caltech for their advice and help.

\section{REFERENCES}

[1] C. Pang, S. Musallam, Y.-C. Tai, J. W. Burdick, and R. A. Andersen, "Novel Monolithic Silicon Probes with Flexible Parylene Cables for Neural Prostheses," IEEE-EMBS MMB, 2006.

[2] W. Li, D.C. Rodger, J.D. Weiland, M.S. Humayun, Y.C. Tai, "Integrated Flexible Ocular Coil for Power and Data Transfer in Retinal Prostheses," Proc. 27th Eng. in Med. and Biol. Soc., 2005

[3] P. Srinivasan, F. R. Beyette, and I. Papautsky , "Micromachined Arrays of Cantilevered Glass Probes," Appl. Opt. 43, 776-782 (2004)

[4] J. Vossen, W. Kern, Thin Film Processes, Academic Press, 1978, p. 440

[5] D. Robinson, "The Electrical Properties of Metal Microelectrodes," Preceedings of the IEEE, Vol. 56, No 6. 1968 